\title{
Efficient Computation of Canonical Form for Boolean Matching in Large Libraries
}

\author{
Debatosh Debnath \\ Dept. of Computer Science \& Engineering \\ Oakland University, Rochester \\ Michigan 48309, U.S.A. \\ debnath@oakland.edu
}

\author{
Tsutomu Sasao \\ Dept. of Computer Science \& Electronics \\ Kyushu Institute of Technology \\ Iizuka 820-8502, JAPAN \\ sasao@cse.kyutech.ac.jp
}

\begin{abstract}
This paper presents an efficient technique for solving a Boolean matching problem in cell-library binding, where the number of cells in the library is large. As a basis of the Boolean matching, we use the notion NP-representative (NPR); two functions have the same NPR if one can be obtained from the other by a permutation and/or complementation(s) of the variables. By using a table look-up and a tree-based breadthfirst search strategy, our method quickly computes NPR for a given function. Boolean matching of the given function against the whole library is determined by checking the presence of its NPR in a hash table, which stores NPRs for all the library functions and their complements. The effectiveness of our method is demonstrated through experimental results, which shows that it is more than two orders of magnitude faster than the Hinsberger-Kolla's algorithm-the fastest Boolean matching algorithm for large libraries.
\end{abstract}

Index Terms-Logic synthesis, Boolean matching, cell-library binding, technology mapping, canonical form.

\section{INTRODUCTION}

Determining whether a circuit can be functionally equivalent to another under a permutation of its inputs, complementation of its one or more inputs, and/or inversion of its output is an important problem in logic synthesis, and Boolean matching technique is used to solve it. Algorithms for Boolean matching have applications in cell-library binding where it is necessary to repeatedly check if some part of a multiplelevel representation of a Boolean function can be realized by any of the cells from a given library [7], in logic verification where correspondence of the inputs of two circuits are unknown [14, 24], and in table look-up based logic synthesis [5]. In this paper, we consider Boolean matching problem for cell-library binding. An exhaustive method for Boolean matching is computationally expensive even for functions with only few variables, because the time complexity of such an algorithm for an $n$-variable function is $O\left(n ! 2^{2 n}\right)$.

Boolean matching phase is one of the most time consuming steps in cell library binding, and because of their importance in synthesizing cost effective circuits, Boolean matching problems received much attention and many algorithms have been developed to efficiently solve them [1].
Signatures, which are computed from some properties of Boolean functions, are extensively used in Boolean matching [1]. Equality in the signatures are a necessary condition for Boolean matching of two functions; although it is not the sufficient condition, signature-based algorithms have successfully demonstrated their effectiveness. Some of the signature-based algorithms are efficient for performing pair-wise Boolean matching [14, 18, 23, 24]; however, to match a function against a library they often require to perform pair-wise matchings of the function with all the library cells. Therefore, Boolean matching techniques based on them are unsuitable for handling libraries with large number of cells. There are other signature-based algorithms that are successfully used with cell libraries; however, they can handle libraries with only modest size $[4,16,22]$. Moreover, due to the lack of sufficient information in the signatures, algorithms based on them in many cases are unable to conclude a Boolean match. Thus, an exhaustive search is necessary to obtain a conclusive result. Some other Boolean matching algorithms consider only some restricted form of Boolean matching $[8,12,20,21]$.

There are other categories of Boolean matching algorithms that are based on the computation of some canonical form for Boolean functions [2, 3, 6, 10, 25]. Two functions match if their canonical forms are the same. The Boolean matching technique that we consider in this paper falls under this category. Burch and Long introduced a canonical form for matching under complementation and a semi-canonical form for matching under permutation of the variables [2]. These two forms can be combined to check Boolean matching under permutation and complementation of variables. However, a large number of forms for each cells are required when using the method in cell library binding. Ciric and Sechen [3], Debnath and Sasao [6], and Wu et al. [25] also proposed canonical forms for efficient Boolean matching; however, their techniques are applicable for Boolean matching under permutation of the variables only. Hinsberger and Kolla introduced a canonical form for solving the general Boolean matching problem that we are considering in this paper [10]. To the best of our knowledge, it is the fastest Boolean matching algorithm that can handle libraries with 
large number of cells under permutation and complementation of the variables as well as inversion of the function; however, the method still requires considerable computation.

In this paper, we present an efficient technique for computing a canonical form for Boolean functions. The canonical form-which we refer to as NP-representative (NPR)remains unchanged under permutation and complementation of the variables. The set of functions that can be made identical under permutation and complementation of the variables form an $N P$-equivalence class $[9,11,19]$. In an NP-equivalence class, the function that has the smallest value in the binary representation is the NPR of the class, and every NP-equivalence class has a unique NPR. Thus, if the NPRs for the functions represented by the two circuits are equal, they are functionally equivalent under the permutation and complementation of inputs. It should be noted that our canonical form is similar to that of Hinsberger and Kolla [10]. For efficient computation of NPRs we use precomputed NP-transformation tables (NPTTs), which are used to quickly generate any functions in an NP-equivalence class. To make the search even more efficient, our method combines an NPTT with a search tree for each variables and performs breadth-first searches.

Although NPRs can identify functional equivalence of two circuits under permutation and complementation of inputs, they are unable to directly ascertain the functional equivalence if it involves determining whether the output of one circuit is also complemented. Thus, to handle the output complementation we use the following strategy. Let $f$ and $g$ be the Boolean functions represented by two circuits $F$ and $G$, respectively. To check if $F$ and $G$ are functionally equivalent under permutation and complementation of inputs of $G$ as well as possible complementation of its output, we compute the NPRs for $f, g$, and $\bar{g}$. If the NPRs for $f$ and $g$ are equal, $G$ can be made functionally equivalent to $F$ by permutation and complementation of inputs of $G$; however, if the NPRs for $f$ and $\bar{g}$ are equal, complementation of the output of $G$ is also required in addition to permutation and complementation of its inputs to make $F$ and $G$ functionally equivalent.

For using our method in cell library binding the library requires to be preprocessed. A set of personalized modules can be obtained from each library cells by bridging some of its inputs and/or setting some of its inputs to constant values [10]. In the preprocessing phase we generate library functions, which are the collection of functions represented by the library cells and by the personalized modules obtained from the library cells.

For Boolean matching of cell libraries we precompute the NPRs for the library functions and their complements. When we require to find a Boolean match of a given function against the whole library, we compute its NPR and check whether the same NPR is present in the precomputed NPRs for the library functions. An affirmative answer indicates a Boolean matching with a cell in the library. For efficient equivalence checking of NPRs we use a hash table to store the NPRs for the library functions.
Based on the above discussions, our Boolean matching technique for library binding can be summarized as:

- Build the breadth-first search trees for each variables.

- Generate the library functions; for each of them, compute two NPRs-one for it and the other for its complement-and store them in a hash table.

- Compute the NPR for the function to be matched against the library.

- Check the hash table for the presence of the NPR; a matching is found if the NPR is in the table.

We will refer to the first two of the above steps as the setup phase and the last two steps as the matching phase.

Usually Boolean matching for libraries with large number of cells is computationally expensive; on the other hand, an increase in the number of the cells in a library often improves the quality of the mapped circuits $[15,21]$. Since pair-wise matchings are unnecessary, the computational complexity of our Boolean matching technique is almost independent of the number of cells in the library. Moreover, our method is independent of any cell architecture and any functional properties. However, functional properties can be used with our method as filters for quickly detecting the functions that cannot be matched against a library $[4,14,16,17,20,22]$. Experimental results and comparison with another method demonstrate that the proposed technique is highly effective.

The remainder of the paper is organized as follows: Section II formally introduces the terminology. Section III develops the techniques for computing the NPR, which is the basis of our Boolean matching technique. Section IV reports experimental results and compares our technique with another method. Section V presents conclusions.

\section{DEFINITIONS AND TERMINOLOGY}

Definition 1 Let the minterm expansion of an n-variable function $f\left(x_{1}, x_{2}, \ldots, x_{n}\right)$ be $m_{0} \cdot \bar{x}_{1} \bar{x}_{2} \cdots \bar{x}_{n} \vee m_{1} \cdot \bar{x}_{1} \bar{x}_{2} \cdots x_{n} \vee$ $\cdots \vee m_{2^{n}} \cdot x_{1} x_{2} \cdots x_{n}$, where $m_{0}, m_{1}, \ldots, m_{2^{n}-1} \in\{0,1\}$. Let the $2^{n}$ bit binary number $m_{0} m_{1} \cdots m_{2^{n}}$, which is obtained by the concatenation of $m_{0}, m_{1}, \ldots, m_{2^{n}-1}$ in this order, be the binary representation of $f$. To denote a binary number, usually a subscripted 2 is used after it.

Example 1 Consider the three-variable function $f\left(x_{1}, x_{2}, x_{3}\right)=\bar{x}_{1} x_{2} x_{3} \vee x_{1} \bar{x}_{2} \bar{x}_{3}$. The binary representation of $f$ is $00011000_{2}$.

Definition 2 Two functions $f$ and $g$ are NP-equivalent if $g$ can be obtained from $f$ by a permutation of the variables and/or complementation of one or more variables [9, 11, 19]. NP-equivalent functions form an NP-equivalence class of functions.

Example 2 Consider the four functions: $f_{1}\left(x_{1}, x_{2}, x_{3}\right)=$ $\bar{x}_{1} \bar{x}_{2} \bar{x}_{3} \vee x_{1} x_{2} x_{3}, \quad f_{2}\left(x_{1}, x_{2}, x_{3}\right)=\bar{x}_{1} \bar{x}_{2} x_{3} \vee x_{1} x_{2} \bar{x}_{3}$, $f_{3}\left(x_{1}, x_{2}, x_{3}\right)=\bar{x}_{1} x_{2} \bar{x}_{3} \vee x_{1} \bar{x}_{2} x_{3}$, and $f_{4}\left(x_{1}, x_{2}, x_{3}\right)=\bar{x}_{1} x_{2} x_{3} \vee$ $x_{1} \bar{x}_{2} \bar{x}_{3}$. Since $f_{2}\left(x_{1}, x_{2}, \bar{x}_{3}\right)=\bar{x}_{1} \bar{x}_{2} \bar{x}_{3} \vee x_{1} x_{2} x_{3}=f_{1}\left(x_{1}, x_{2}, x_{3}\right)$, 
$f_{1}$ and $f_{2}$ are NP-equivalent. Similarly, we can show that $f_{3}$ and $f_{4}$ are also NP-equivalent to $f_{1}$. Therefore, the functions $f_{1}, f_{2}, f_{3}$, and $f_{4}$ belong to the same NP-equivalence class.

Definition 3 The function that has the smallest value in the binary representation among the functions of an $N P$-equivalence class is the NP-representative (NPR) of that class.

Example 3 From Example 2, all the functions of an NP-equivalence class are $\bar{x}_{1} \bar{x}_{2} \bar{x}_{3} \vee x_{1} x_{2} x_{3}, \bar{x}_{1} \bar{x}_{2} x_{3} \vee x_{1} x_{2} \bar{x}_{3}$, $\bar{x}_{1} x_{2} \bar{x}_{3} \vee x_{1} \bar{x}_{2} x_{3}$, and $\bar{x}_{1} x_{2} x_{3} \vee x_{1} \bar{x}_{2} \bar{x}_{3}$. In binary representation: $\bar{x}_{1} \bar{x}_{2} \bar{x}_{3} \vee x_{1} x_{2} x_{3}=10000001_{2}, \bar{x}_{1} \bar{x}_{2} x_{3} \vee x_{1} x_{2} \bar{x}_{3}=$ $01000010_{2}, \bar{x}_{1} x_{2} \bar{x}_{3} \vee x_{1} \bar{x}_{2} x_{3}=00100100_{2}$, and $\bar{x}_{1} x_{2} x_{3} \vee$ $x_{1} \bar{x}_{2} \bar{x}_{3}=00011000_{2}$. Since $00011000_{2}<00100100_{2}<$ $01000010_{2}<10000001_{2}$, the NP-representative of the class is $\bar{x}_{1} x_{2} x_{3} \vee x_{1} \bar{x}_{2} \bar{x}_{3}$.

Variables of an $n$-variable function can be permuted in $n$ ! ways and complemented in $2^{n}$ ways; thus the total number of possible combinations are $n ! 2^{n}$. However, for many functions some of these combinations generate the same function. Therefore, for an $n$-variable function there are at most $n ! 2^{n}$ NP-equivalents. Among them, our objective is to quickly find the NP-equivalent that has the smallest value in the binary representation.

\section{COMPUTING NP-REPRESENTATIVE}

In this section, we show a method to compute NP-representative (NPR) by using three-variable functions and discuss how the technique can be extended to functions with more variables.

\subsection{Binary Representations Under Permutation and Com- plementation of Variables}

Binary representations of a given function under different permutation and complementation of variables can be easily generated if the function is represented as minterm expansion. For example, let

$$
\begin{aligned}
f\left(x_{1}, x_{2}, x_{3}\right)= & m_{0} \bar{x}_{1} \bar{x}_{2} \bar{x}_{3} \vee m_{1} \bar{x}_{1} \bar{x}_{2} x_{3} \vee \\
& m_{2} \bar{x}_{1} x_{2} \bar{x}_{3} \vee m_{3} \bar{x}_{1} x_{2} x_{3} \vee \\
& m_{4} x_{1} \bar{x}_{2} \bar{x}_{3} \vee m_{5} x_{1} \bar{x}_{2} x_{3} \vee \\
& m_{6} x_{1} x_{2} \bar{x}_{3} \vee m_{7} x_{1} x_{2} x_{3}
\end{aligned}
$$

be the minterm expansion of a three-variable function, where $m_{0}, m_{1}, \ldots, m_{7} \in\{0,1\}$. The permutation of the variables in (1) is $\left(x_{1}, x_{2}, x_{3}\right)$. When the permutation of the variables is $\left(x_{3}, x_{2}, x_{1}\right)$, we have

$$
\begin{aligned}
f\left(x_{3}, x_{2}, x_{1}\right)= & m_{0} \bar{x}_{3} \bar{x}_{2} \bar{x}_{1} \vee m_{1} \bar{x}_{3} \bar{x}_{2} x_{1} \vee \\
& m_{2} \bar{x}_{3} x_{2} \bar{x}_{1} \vee m_{3} \bar{x}_{3} x_{2} x_{1} \vee \\
& m_{4} x_{3} \bar{x}_{2} \bar{x}_{1} \vee m_{5} x_{3} \bar{x}_{2} x_{1} \vee \\
& m_{6} x_{3} x_{2} \bar{x}_{1} \vee m_{7} x_{3} x_{2} x_{1} \\
= & m_{0} \bar{x}_{1} \bar{x}_{2} \bar{x}_{3} \vee m_{4} \bar{x}_{1} \bar{x}_{2} x_{3} \vee \\
& m_{2} \bar{x}_{1} x_{2} \bar{x}_{3} \vee m_{6} \bar{x}_{1} x_{2} x_{3} \vee \\
& m_{1} x_{1} \bar{x}_{2} \bar{x}_{3} \vee m_{5} x_{1} \bar{x}_{2} x_{3} \vee \\
& m_{3} x_{1} x_{2} \bar{x}_{3} \vee m_{7} x_{1} x_{2} x_{3} .
\end{aligned}
$$

\begin{tabular}{|c|c|c|c|}
\hline$f\left(x_{1}, x_{2}, x_{3}\right)$ & $f\left(x_{1}, \bar{x}_{3}, x_{2}\right)$ & $f\left(\bar{x}_{3}, x_{2}, \bar{x}_{1}\right)$ & $f\left(\bar{x}_{2}, \bar{x}_{1}, x_{3}\right)$ \\
\hline$m_{0}$ & $m_{2}$ & $m_{5}$ & $m_{6}$ \\
$m_{1}$ & $m_{0}$ & $m_{1}$ & $m_{7}$ \\
$m_{2}$ & $m_{3}$ & $m_{7}$ & $m_{2}$ \\
$m_{3}$ & $m_{1}$ & $m_{3}$ & $m_{3}$ \\
$m_{4}$ & $m_{6}$ & $m_{4}$ & $m_{4}$ \\
$m_{5}$ & $m_{4}$ & $m_{0}$ & $m_{5}$ \\
$m_{6}$ & $m_{7}$ & $m_{6}$ & $m_{0}$ \\
$m_{7}$ & $m_{5}$ & $m_{2}$ & $m_{1}$ \\
\hline
\end{tabular}

Figure 1: Four of the NP-equivalents of a three-variable function $f\left(x_{1}, x_{2}, x_{3}\right)$.

From (1) and (2), the binary representations of $f\left(x_{1}, x_{2}, x_{3}\right)$ and $f\left(x_{3}, x_{2}, x_{1}\right)$ are $m_{0} m_{1} m_{2} m_{3} m_{4} m_{5} m_{6} m_{7}$ and $m_{0} m_{4} m_{2} m_{6} m_{1} m_{5} m_{3} m_{7}$, respectively.

When variables are complemented, the binary representation can also be generated in a similar manner. For example, by replacing $x_{1}$ by $\bar{x}_{1}$ in (2), we have

$$
\begin{aligned}
f\left(x_{3}, x_{2}, \bar{x}_{1}\right)= & m_{0} x_{1} \bar{x}_{2} \bar{x}_{3} \vee m_{4} x_{1} \bar{x}_{2} x_{3} \vee \\
& m_{2} x_{1} x_{2} \bar{x}_{3} \vee m_{6} x_{1} x_{2} x_{3} \vee \\
& m_{1} \bar{x}_{1} \bar{x}_{2} \bar{x}_{3} \vee m_{5} \bar{x}_{1} \bar{x}_{2} x_{3} \vee \\
& m_{3} \bar{x}_{1} x_{2} \bar{x}_{3} \vee m_{7} \bar{x}_{1} x_{2} x_{3} \\
= & m_{1} \bar{x}_{1} \bar{x}_{2} \bar{x}_{3} \vee m_{5} \bar{x}_{1} \bar{x}_{2} x_{3} \vee \\
& m_{3} \bar{x}_{1} x_{2} \bar{x}_{3} \vee m_{7} \bar{x}_{1} x_{2} x_{3} \vee \\
& m_{0} x_{1} \bar{x}_{2} \bar{x}_{3} \vee m_{4} x_{1} \bar{x}_{2} x_{3} \vee \\
& m_{2} x_{1} x_{2} \bar{x}_{3} \vee m_{6} x_{1} x_{2} x_{3},
\end{aligned}
$$

which gives $m_{1} m_{5} m_{3} m_{7} m_{0} m_{4} m_{2} m_{6}$ as the binary representation of $f\left(x_{3}, x_{2}, \bar{x}_{1}\right)$. Several randomly chosen NP-equivalents of $f\left(x_{1}, x_{2}, x_{3}\right)$ are shown in Fig. 1, where the binary representations are written vertically; in the subsequent discussions, binary representations will often be displayed in this way.

\subsection{Basic Idea}

Fig. 1 shows four of the NP-equivalents of $f\left(x_{1}, x_{2}, x_{3}\right)$. There are at most $48\left(=3 ! 2^{3}\right)$ NP-equivalents of a threevariable function. Our objective in computing the NPR is to find the NP-equivalent that has the smallest value in the binary representation. Thus, we can generate NP-equivalents with other permutations and complementations of the variables, and take the function that has the smallest value in the binary representation as the NPR.

An observation to the minterms of the first and second columns of Fig. 1 shows that all the minterms of $f\left(x_{1}, x_{2}, x_{3}\right)$ move to new positions in $f\left(x_{1}, \bar{x}_{3}, x_{2}\right)$. For example, the first minterm, $m_{0}$, of $f\left(x_{1}, x_{2}, x_{3}\right)$ becomes the second minterm of $f\left(x_{1}, \bar{x}_{3}, x_{2}\right)$. Therefore, each time we want to change the permutation and complementation of the variables of an $n$-variable function, we must compute the new positions for all the $2^{n}$ minterms. Since an $n$-variable function has at most $n ! 2^{n}$ NP-equivalents, to compute the NPR for an $n$ variable function we must compute $n ! 2^{2 n}$ new positions for 
TABLE I

MAXIMUM Number of NP-EQUiVALENTS AND Size OF NPTTS FOR DIFFERENT NUMBER OF VARIABLES

\begin{tabular}{|c|c|c|}
\hline $\begin{array}{c}\text { Number of } \\
\text { variables }\end{array}$ & $\begin{array}{c}\text { Maximum number } \\
\text { of NP-equivalents }\end{array}$ & $\begin{array}{c}\text { Size of } \\
\text { NPTT }\end{array}$ \\
\hline 3 & 48 & 384 \\
\hline 4 & 384 & 6144 \\
\hline 5 & 3840 & 122880 \\
\hline 6 & 46080 & 2949120 \\
\hline 7 & 645120 & 82575360 \\
\hline 8 & 10321920 & $2.64 \times 10^{9}$ \\
\hline
\end{tabular}

the minterms, i.e., the time complexity of the algorithm is $O\left(n ! 2^{2 n}\right)$. As a result the method requires significant amount of computation time even for functions with as few as three variables.

\subsection{NP-Transformation Table (NPTT)}

Fig. 1 shows that the new positions of the minterms are fixed for each of the permutation and complementation of the variables. Therefore, our strategy is to compute the new positions of the minterms for all the permutation and complementation of the variables only once and to use them repeatedly for computing NPRs; this method is much faster than the method presented in Section 3.2, because repeated computation of the new positions for the minterms is unnecessary. Fig. 2 shows a table of all such new positions of the minterms for three-variable function; it is similar to Fig. 1 except column headings are removed and $m_{i}$ is replaced by $i(0 \leq i \leq 7)$. We will refer to such a table as NP-transformation table (NPTT). Although column headings are removed from Fig. 2 for ease of showing the whole table, they are required by our algorithm.

The NPTT for an $n$-variable function has $2^{n}$ rows and $n ! 2^{n}$ columns, i.e., it has $n ! 2^{2 n}$ entries (Fig. 2). Table I shows the maximum number of NP-equivalents in an NP-equivalence class and the size of NPTTs for different number of variables. Since the size of the NPTTs grow exponentially, they can be practically used for functions with up to seven variables, which is the upper bound on the variables for which our Boolean matching technique can be applicable. It should be noted that the maximum number of inputs to the cells in many cell libraries is less than seven.

\subsection{Breadth-First Search by Using NPTT}

The straightforward method for computing NPR by using NPTT that we presented in Section 3.3 first generates all the $n ! 2^{n}$ NP-equivalents from a given $n$-variable function, and then chooses one with the smallest value in the binary representation as the NPR. Since we are interested only in the function that has the smallest value in the binary representation, we may avoid generating many of the NP-equivalents. Each columns of the NPTT corresponds to an NP-equivalent, and we use a breadth-first search technique to early detect the columns that cannot lead to the NPR. In this method, the row at the top of the NPTT is used at first to generate the first minterms of all the NP-equivalents, where the first minterm is the left most minterm in the binary representation. After generating the first minterms corresponding to all the columns of the NPTT, we apply the following:

(a) if the minterms have both 0 and 1 values, we only keep the columns that generate minterms with only 0 value and discard other columns, and

(b) if all the minterms have either 0 or 1 values, we keep all the columns.

Since NPR has the smallest value in the binary representation among all the NP-equivalents, step (a) effectively discards some of the columns that cannot lead to the NPR. We then use second row of the NPTT for generating the second minterms correspond to the columns that we kept in steps (a) and (b), and apply steps (a) and (b) on the second minterms for possibly discarding some of the remaining columns from consideration. We continue this process until the bottom row of the NPTT is considered. At this point search terminates, and any of the remaining columns can generate an NPR. It should be noted that the breadth-first search technique is difficult to apply if we cannot store NPTT.

\subsection{Combining NPTT with a Breadth-First Search Tree}

Although the breadth-first search by using NPTT can reduce the search space quickly, by combining a search tree with the NPTT we can make the search even more efficient. In Section 3.4, NPTT is used row by row for computing NPR. The breadth-first search by using NPTT in Fig. 2 requires first to check all the 48 elements on the first row. An observation to Fig. 2 shows that there are 8 distinct elements on the first row. Therefore, we can partition these elements in to 8 groups and perform 8 checks-instead of 48 - to determine any columns that cannot lead to NPR; in this case also we use the breadth-first search strategy that are used in steps (a) and (b) of Section 3.4; but the number of checks here is only 8 .

Fig. 2 also shows that each of these groups can be partitioned in to 3 subgroups, which in turn can be again partitioned in to 2 subgroups. These lead to a breadth-first search tree shown in Fig. 3, where the branches of the tree are labeled with the elements of the first three rows of the NPTT. Fig. 3 shows that the top three rows of Fig. 2 form the search tree, while the bottom five rows stay the same. Therefore, the search for an NPR starts at the root of the search tree; after reaching the bottom of the tree the search continues from the fourth row of the NPTT until its bottom row is considered.

The breadth-first search tree for an $n$-variable function can be constructed in a similar manner. The root node of the search tree has $2^{n}$ children; the number of children for each of the nodes in the subsequent levels has $n, n-1, \ldots, 3$, and 2 children. 
$\begin{array}{llllllllllllllllllllllllllllllllllllllllllllllll}0 & 0 & 0 & 0 & 0 & 0 & 1 & 1 & 1 & 1 & 1 & 1 & 2 & 2 & 2 & 2 & 2 & 2 & 3 & 3 & 3 & 3 & 3 & 3 & 4 & 4 & 4 & 4 & 4 & 4 & 5 & 5 & 5 & 5 & 5 & 5 & 6 & 6 & 6 & 6 & 6 & 6 & 7 & 7 & 7 & 7 & 7 & 7\end{array}$ $\begin{array}{lllllllllllllllllllllllllllllllllllllllllllllllll}1 & 1 & 2 & 2 & 4 & 4 & 0 & 0 & 3 & 3 & 5 & 5 & 0 & 0 & 3 & 3 & 6 & 6 & 1 & 1 & 2 & 2 & 7 & 7 & 0 & 0 & 5 & 5 & 6 & 6 & 1 & 1 & 4 & 4 & 7 & 7 & 2 & 2 & 4 & 4 & 7 & 7 & 3 & 3 & 5 & 5 & 6 & 6\end{array}$ $\begin{array}{llllllllllllllllllllllllllllllllllllllllllllllll}2 & 4 & 1 & 4 & 1 & 2 & 3 & 5 & 0 & 5 & 0 & 3 & 3 & 6 & 0 & 6 & 0 & 3 & 2 & 7 & 1 & 7 & 1 & 2 & 5 & 6 & 0 & 6 & 0 & 5 & 4 & 7 & 1 & 7 & 1 & 4 & 4 & 7 & 2 & 7 & 2 & 4 & 5 & 6 & 3 & 6 & 3 & 5\end{array}$ $\begin{array}{lllllllllllllllllllllllllllllllllllllllllllllllll}3 & 5 & 3 & 6 & 5 & 6 & 2 & 4 & 2 & 7 & 4 & 7 & 1 & 4 & 1 & 7 & 4 & 7 & 0 & 5 & 0 & 6 & 5 & 6 & 1 & 2 & 1 & 7 & 2 & 7 & 0 & 3 & 0 & 6 & 3 & 6 & 0 & 3 & 0 & 5 & 3 & 5 & 1 & 2 & 1 & 4 & 2 & 4\end{array}$ $\begin{array}{lllllllllllllllllllllllllllllllllllllllllllllllll}4 & 2 & 4 & 1 & 2 & 1 & 5 & 3 & 5 & 0 & 3 & 0 & 6 & 3 & 6 & 0 & 3 & 0 & 7 & 2 & 7 & 1 & 2 & 1 & 6 & 5 & 6 & 0 & 5 & 0 & 7 & 4 & 7 & 1 & 4 & 1 & 7 & 4 & 7 & 2 & 4 & 2 & 6 & 5 & 6 & 3 & 5 & 3\end{array}$ $\begin{array}{llllllllllllllllllllllllllllllllllllllllllllllll}5 & 3 & 6 & 3 & 6 & 5 & 4 & 2 & 7 & 2 & 7 & 4 & 4 & 1 & 7 & 1 & 7 & 4 & 5 & 0 & 6 & 0 & 6 & 5 & 2 & 1 & 7 & 1 & 7 & 2 & 3 & 0 & 6 & 0 & 6 & 3 & 3 & 0 & 5 & 0 & 5 & 3 & 2 & 1 & 4 & 1 & 4 & 2\end{array}$ $\begin{array}{llllllllllllllllllllllllllllllllllllllllllllllll}6 & 6 & 5 & 5 & 3 & 3 & 7 & 7 & 4 & 4 & 2 & 2 & 7 & 7 & 4 & 4 & 1 & 1 & 6 & 6 & 5 & 5 & 0 & 0 & 7 & 7 & 2 & 2 & 1 & 1 & 6 & 6 & 3 & 3 & 0 & 0 & 5 & 5 & 3 & 3 & 0 & 0 & 4 & 4 & 2 & 2 & 1 & 1\end{array}$

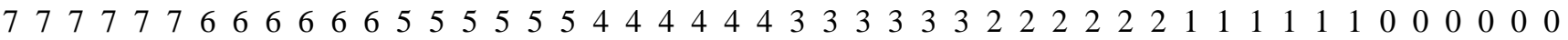

Figure 2: NP-transformation table (NPTT) for three variables.

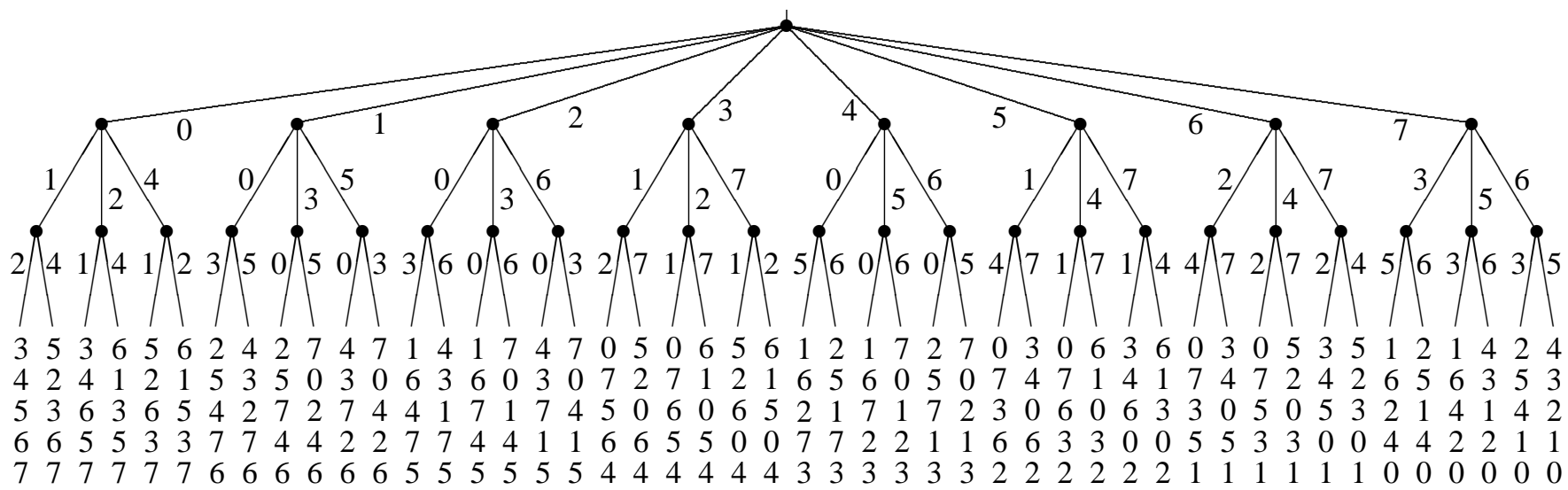

Figure 3: Breadth-first search tree combined with partial NPTT for three variables.

\section{EXPERIMENTAL RESULTS}

We implemented the proposed Boolean matching technique for functions with up to seven variables on a Sun Fire 280R Server. The program requires about 140 megabytes memory, of which about 85 megabytes are used to store the NP-transformation tables (NPTTs); the data structure of the breadth-first search trees and the code of the program use the remaining 55 megabytes. It should be noted that additional memory is required to store NP-representatives (NPRs) for the library functions and their associated hash tables; however, this memory requirement is relatively lower as every byte of memory can hold up to eight bits of the binary representation of NPRs. During setup phase, the program constructs the breadth-first search trees; it takes about $0.50 \mathrm{sec}-$ onds.

To demonstrate the effectiveness of our technique, we conducted an experiment by using 5,000,000 pseudo-random functions with three to seven variables and tried to match them against a cell library, which is represented by 50,000 randomly generated library functions. Table II summarizes the average Boolean matching time in microseconds, which is the time required to match a function against the entire library whose cells generate 50,000 library functions. We note that Boolean matching time of our algorithm is almost independent of whether or not a matching is found.

Hinsberger and Kolla reported Boolean matching time for their TEMPLATE technology mapping system in [10]. From multiple-level networks of NOR gates, TEMPLATE generates
TABLE II

Average Time for Boolean Matching

\begin{tabular}{|c|c|}
\hline $\begin{array}{c}\text { Number of } \\
\text { variables }\end{array}$ & $\begin{array}{c}\text { Time } \\
\text { (microseconds) }\end{array}$ \\
\hline 3 & 9.81 \\
\hline 4 & 15.74 \\
\hline 5 & 26.02 \\
\hline 6 & 39.13 \\
\hline 7 & 147.61 \\
\hline
\end{tabular}

all possible single-output cluster functions [7] with six and fewer variables [13]. It then tries to find a Boolean match for each of the cluster functions against the lib2 library from the MCNC. In library binding of a set of 18 benchmark functions by using lib2 - which consists of 27 cells-TEMPLATE checks total 113,188 Boolean matchings in 9,141 seconds on an HP 735/125, i.e., on the average 12.38 matching attempts per second.

Since experimental results for both the systems are unavailable in the same format, a comparison of the speed performance of our Boolean matching technique with that of the Template is not straightforward. We consider that a Sun Fire 280R Server $(900-\mathrm{MHz}$ UltraSPARC-III processor) is about eight times faster than an HP 735/125 (usually $125-\mathrm{MHz}$ PA-7150 RISC processor). Thus, if all the cluster functions generated by TEMPLATE depends on four, five, and six variables, our method is about 600,400 , and 250 times, respectively, faster than TEMPLATE. In practice none of 
these assumptions about the distribution of the cluster functions could be true, and the actual speed-up would probably be in between these numbers.

\section{Conclusions And Comments}

Fast algorithms for Boolean matching can significantly speed-up the cell library binding process, and Boolean matching for cell library binding where the library contains large number of cells can considerably improve the quality of the solutions. We used the notion NP-representative (NPR) which is unique for any NP-equivalence classes, and presented a table look-up based breadth-first search algorithm to quickly compute it; we used NPRs to efficiently check the functional equivalence of a given circuit against a large library under permutation and complementation of inputs and complementation of output. The method is more than two orders of magnitude faster than Hinsberger-Kolla's algorithm [10].

Our technique is practical for functions with up to seven variables; this number is sufficiently large to work with many cell libraries such as lib2, which consists of cells with up to six inputs and is extensively used by the research community. Although the memory usage of our method is relatively higher than most other Boolean matching algorithms, we believe its superior speed performance and the ability to handle large libraries would outweigh any considerations for its memory requirement. The data structure of our system is only partially optimized, and there are scope for improvement in both memory usage and speed performance.

\section{ACKNOWLEDGEMENT}

We thank Professor Reiner Kolla for interesting discussions about Boolean matching in TEMPLATE system.

\section{REFERENCES}

[1] L. Benini and G. De Micheli, "A survey of Boolean matching techniques for library binding," ACM Trans. Design Automation of Electronic Systems, vol. 2, no. 3, pp. 193-226, July 1997.

[2] J. R. Burch and D. E. Long, "Efficient Boolean function matching," in Proc. IEEE/ACM Int. Conf. on Computer-Aided Design, pp. 408-411, Nov. 1992.

[3] J. Ciric and C. Sechen, "Efficient canonical form for Boolean matching of complex functions in large libraries," IEEE Trans. Computer-Aided Design of Integrated Circuits and Systems, vol. 22, no. 5, pp. 535-544, May 2003.

[4] E. M. Clarke, K. L. McMillan, X. Zhao, M. Fujita, and J. Yang, "Spectral transforms for large Boolean functions with applications to technology mapping," Formal Methods in System Design: An Int. Journal, vol. 10, no. 2, pp. 137-148, Apr. 1997.

[5] D. Debnath and T. Sasao, "A heuristic algorithm to design AND-OR-EXOR three-level networks," in Proc. Asia and South Pacific Design Automation Conf., pp. 69-74, Feb. 1998.
[6] D. Debnath and T. Sasao, "Fast Boolean matching under permutation using representative," in Proc. Asia and South Pacific Design Automation Conf., pp. 359-362, Jan. 1999.

[7] G. De Micheli, Synthesis and Optimization of Digital Circuits, McGraw-Hill, 1994.

[8] S. Ercolani and G. De Micheli, "Technology mapping for electrically programmable gate arrays," in Proc. IEEE/ACM Design Automation Conf., pp. 234-239, June 1991.

[9] M. A. Harrison, Introduction to Switching and Automata Theory, McGraw-Hill, 1965.

[10] U. Hinsberger and R. Kolla, "Boolean matching for large libraries," in Proc. IEEE/ACM Design Automation Conf., pp. 206-211, June 1998.

[11] S. L. Hurst, D. M. Miller, and J. C. Muzio, Spectral Techniques in Digital Logic, Academic Press Inc., 1985.

[12] M. Hütter and M. Scheppler, "Memory efficient and fast Boolean matching for large functions using rectangle representation," in IEEE/ACM Int. Workshop on Logic Synthesis, May 2003.

[13] R. Kolla, "Personal communication,” June 2003.

[14] Y.-T. Lai, S. Sastry, and M. Pedram, "Boolean matching using binary decision diagrams with applications to logic synthesis and verification," in Proc. IEEE Int. Conf. on Computer Design, pp. 452-458, Oct. 1992.

[15] C. Liem and M. Lefebvre, "Performance directed technology mapping using constructive matching," in IEEE/ACM Int. Workshop on Logic Synthesis, May 1991.

[16] F. Mailhot and G. De Micheli, "Algorithms for technology mapping based on binary decision diagrams and on Boolean operations," IEEE Trans. Computer-Aided Design of Integrated Circuits and Systems, vol. 12, no. 5, pp. 599-620, May 1993.

[17] Y. Matsunaga, "A new algorithm for Boolean matching utilizing structural information," IEICE Trans. Information and Systems, vol. E78-D, no. 3, pp. 219-223, Mar. 1995.

[18] J. Mohnke and S. Malik, "Permutation and phase independent Boolean comparison," in Proc. IEEE European Conf. on Design Automation, pp. 86-92, Feb. 1993.

[19] S. Muroga, Logic Design and Switching Theory, John Wiley \& Sons, 1979.

[20] U. Schlichtmann, F. Brglez, and M. Hermann, "Characterization of Boolean functions for rapid matching in EPGA technology mapping," in Proc. IEEE/ACM Design Automation Conf., pp. 374-379, June 1992.

[21] U. Schlichtmann, F. Brglez, and P. Schneider, "Efficient Boolean matching based on unique variable ordering," in IEEE/ACM Int. Workshop on Logic Synthesis, pp. 3b:1-3b:13, May 1993.

[22] E. Schubert and W. Rosenstiel, "Combined spectral techniques for Boolean matching," in Proc. ACM Int. Symposium on Field-Programmable Gate Arrays, pp. 38-43, Feb. 1996.

[23] C. Tsai and M. Marek-Sadowska, "Boolean functions classification via fixed polarity Reed-Muller forms," IEEE Trans. Comput., vol. 46, no. 2, pp. 173-186, Feb. 1997.

[24] K.-H. Wang, T. Hwang, and C. Chen, "Exploiting communication complexity for Boolean matching," IEEE Trans. Computer-Aided Design of Integrated Circuits and Systems, vol. 15, no. 10, pp. 1249-1256, Oct. 1996.

[25] Q. Wu, C. Y. R. Chen, and J. M. Acken, "Efficient Boolean matching algorithm for cell libraries," in Proc. IEEE Int. Conf. on Computer Design, pp. 36-39, Oct. 1994. 